Check for updates

Cite this: RSC Adv., 2017, 7, 32008

Received 21st February 2017

Accepted 14th June 2017

DOI: $10.1039 / c 7 r a 02171 b$

rsc.li/rsc-advances

\section{Cost-effective fabrication of graphene-like nanosheets from natural microcrystalline graphite minerals by liquid oxidation-reduction method $\uparrow$}

\author{
Wei Xie, (D) *abcd Xukun Zhu, ${ }^{\text {ab }}$ Shang Xu, ${ }^{\text {ab }}$ Shihe Yi, ${ }^{c}$ Zhanhu Guo, (D) d Jiacai Kuang ${ }^{\text {ab }}$ \\ and Yingjun Deng ${ }^{a}$
}

It is a challenge to prepare graphene-like nanosheets (GNs) with desired electrical properties from natural microcrystalline graphite minerals (NMGM) since the impurities in NMGM are always hard to eliminate and the grain size of NMGM is much smaller than that of flake graphite (FG). The morphology, structure, surface chemistry, and compositions of the GNs prepared from NMGM using a liquid oxidation-reduction approach were investigated in this study. GNs with layer number ranging from 3 to 7 , thickness of $1.1 \mathrm{~nm}$, and sizes up to $1 \mu \mathrm{m} \times 2 \mu \mathrm{m}$ were obtained and confirmed from scanning electron microscopy, transmission electron microscopy and atomic force microscopy images. The results of Fourier transform infrared spectroscopy and X-ray photoelectron spectroscopy analysis show that, during the whole fabrication process, the generation and elimination of oxygen-containing groups or reestablishment of the conjugated graphene network occur. The results of Raman spectroscopy and X-ray diffraction measurements show that the GNs possess an amorphous nanostructure. The as-produced GNs with adjustable electrical resistivity can be easily transferred into well distributed bulk materials by freeze-dried strategy. This cost-effective synthesis strategy of GNs from abundant NMGM provides great potential for the commercialization of GN production.

\section{Introduction}

Graphene is a monolayer of carbon atoms in a closely packed honeycomb two-dimensional (2D) lattice, and has attracted tremendous scientific attention in recent years. Especially this 2D material exhibits unique physical, chemical, electrical and mechanical properties, which make graphene and its derivatives promising for potential applications in many technological fields, such as chemical screening, sensors, low density structural materials, quantum effects, thermal interface materials, electronic materials, energy storage and energy conversion..$^{1-3}$ Graphene can be prepared through numerous methods, such as chemical oxidation reduction, mechanical exfoliation, thermal exfoliation of graphite oxide, liquid-phase exfoliation

${ }^{a}$ Hunan Province Key Laboratory of Safety Design and Reliability Technology for Engineering Vehicle, Changsha University of Science \& Technology, Changsha 410114, China. E-mail: cslggncl@163.com

${ }^{b}$ Hunan Province Higher Education Key Laboratory of Modeling and Monitoring on the Near-Earth Electromagnetic Environments, Changsha University of Science \& Technology, Changsha 410114, China

'College of Aerospace Science and Engineering, National University of Defense Technology, Changsha 410073, China

${ }^{d}$ Chemical and Biomolecular Engineering Department, University of Tennessee, Knoxville, TN 37996, USA

† Electronic supplementary information (ESI) available. See DOI: $10.1039 / \mathrm{c} 7 \mathrm{ra02171b}$ and supercritical $\mathrm{CO}_{2}$ processing techniques. ${ }^{4-10}$ Among the various methods, chemical reduction of graphene oxide (GO) to reduced graphene oxide (RGO) is unique and attractive because of its capability of producing single-layer graphene in a large scale and at a relatively low cost. Furthermore, GO and RGO are processible and they can be fabricated or self-assembled into macroscopic materials with controlled compositions and microstructures for practical applications. ${ }^{11-14}$

Current commercialized graphene fabricated by chemical oxidation reduction is mainly produced from natural graphite, which is found widely throughout the world and is a kind of frequently used natural material. On earth there is a massive storage of natural graphite minerals, including flake graphite (FG) and microcrystalline graphite (MG). The grain size of FG is usually larger than $10 \mu \mathrm{m}$, however, the concentration of carbon is very low. In contrast, the MG usually has a small grain size of $<1 \mu \mathrm{m}$ but a high carbon content. There are large and widespread deposits of natural microcrystalline graphite minerals (NMGM) in China. The largest mine, with a reserve of 30 million tons, is located in the Lutang area of Chenzhou, Hunan province. Other major deposits are known in the provinces of Inner Mongolia (Bayan Nur), Hubei (Shiyan), Fujian (Sanming), Jilin (Panshi), and Heilongjiang (Yichun). The grade of some European deposits is about $55 \%$, while the counterpart of some MG deposits in China is $50-85 \% .^{15,16}$ 
However, NMGM was inefficiently utilized and seriously wasted due to the low-level technical process. The utilization of NMGM is currently confined to a preliminary level such as raw materials for pencil lead, crucible, and carburant for steel making. Investigations into further applications of NMGM will greatly promote the development of the MG industry. Recently MG has been applied as anode material of lithium-ion battery, refractory, supercapacitor, isotropic graphite and microwave absorbing materials as a cheap and abundant resources. ${ }^{17-26}$ Therefore, a large number of submicron or nano-carbon sources with high processability, large surface area, high activity, and reasonable electrical conductivity should be developed to meet the demand of the industrial applications aforementioned.

Up to now, the research on preparation of graphene-like nanosheets (GNs) is mainly related to FG, a kind of frequently used natural material. However, NMGM, which is a type of resourceful and cheap natural graphite with high degree of graphitization and consists of many microcrystals of different orientations, should be a potential raw material to produce GNs, however, there's few research has been conducted on preparation of GNs from NMGM. The main reason is that the impurities in NMGM are always hard to be eliminated and the grain size of MG is much smaller than that of FG. The carbon content of NMGM can be higher than $99 \%$ as the development of the purification technology goes on, and the smaller grain size of NMGM may lead to higher reactivity, so MG should be oxidized more easily than FG and graphene can be fabricated from NMGM. Fortunately, a few studies on the preparation of GNs by NMGM have initiated. Wang Junying and coworkers produced high-quality GNs at a high yield of $>70 \%$ through a scalable electrochemical \& mechanical exfoliation approach using NMGM directly. ${ }^{27}$ Xian Haiyang and coworkers prepared GNs from MG by a low-temperature exfoliation method, and the supercapacitive behaviors of the GNs have also been investigated. ${ }^{28,29}$ Lin Shaofen and coworkers reported a facile and effective technique for the large-scale production of nano-sized graphene sheets via subjecting the MG to ball milling. The products consist of single- or few-layer graphene with lateral dimensions concentrated in the range of 100-200 nm. ${ }^{30}$ Among them, explanations on the decrease of impurities within NMGM as well as the transformation of both structure and composition of GNs have not been found. However, methods of liquid oxidation-reduction can compensate the disadvantages of methods including electrochemical \& mechanical exfoliation, low-temperature exfoliation and ball milling due to the ease of eliminating the impurities deposited in the raw materials. Furthermore, the fabrication of graphene via liquid oxidationreduction methods is the most promising approach towards large scale production.
Mass production of GNs from graphite at a low cost and a high efficiency is essential for its practical application since there is huge storage of natural graphite minerals on earth. To the best of our knowledge, most of the GNs presented were obtained based on the FG instead of NMGM since it has great difficulty removing the impurities of NMGM through purification technology. Therefore, if the low-cost NMGM can directly be utilized for preparing GNs with good performance and without going through the purification treatment from graphite minerals, it can provide an economic way to realize the comprehensive utilization and widely usage of NMGM. The aim of this study is to use a cost-effective method to prepare graphene with microcrystalline graphite mineral resources that are of low utilization rate, low level of deep processing technology but rich reserves, which will effectively improve the additional value of microcrystalline graphite and provide the basis for the utilization for high value of microcrystalline graphite. And the waste can be changed into resources. In the present work, we prepared GNs from NMGM by liquid oxidation-reduction method, and the microstructure, morphology, layers and compositions of the as-prepared GNs have also been investigated and the formation mechanisms for the liquid oxidationreduction of GNs from NMGM was also discussed.

\section{Experimental}

\subsection{Chemicals and materials}

NMGM (chemical compositions presented in Table 1) with approximately $83.08 \mathrm{wt} \%$ fixed carbon content was used as raw material, obtained from Chenzhou, Hunan province, China. ${ }^{\mathbf{1 6}}$ Concentrated sulfuric acid $\left(\mathrm{H}_{2} \mathrm{SO}_{4}, 98 \%\right)$, potassium permanganate $\left(\mathrm{KMnO}_{4}\right)$, hydrochloric acid $(\mathrm{HCl}, 36 \%)$, sodium nitrate $\left(\mathrm{NaNO}_{3}\right)$ and hydrogen peroxide $\left(\mathrm{H}_{2} \mathrm{O}_{2}, 30 \%\right)$ were purchased from Hengyang Kaixin Chemical Reagent Co. Ltd. Sodium borohydride $\left(\mathrm{NaBH}_{4}\right)$, sodium hydroxide $(\mathrm{NaOH})$ and polyvinylpyrrolidone (PVP) were provided by Guangfu Fine Chemical Research Institute, Fengchuan Chemical Reagent Technology Co. Ltd. and Aladdin Industrial Corporation respectively. Distilled water was processed by our lab. These chemicals were used without further purification.

\subsection{Fabrication of GNs}

Large pieces of NMGM were crushed and dry-grounded by jaw crusher. The ground mineral particles were dry-screened using the standard sieve (200 mesh) and the NMGM with narrow particle size distribution were used as reagents in the chemical fabrication process. There are three stages in the fabrication process of GNs from NMGM by liquid oxidation-reduction method, including the oxidation of NMGM, the stripping of natural microcrystalline graphite oxide and the reduction of

Table 1 Chemical compositions of NMGM (wt\%)

\begin{tabular}{lcccccccrrr}
\hline Index & $\mathrm{C}$ & $\mathrm{SiO}_{2}$ & $\mathrm{Al}_{2} \mathrm{O}_{3}$ & $\mathrm{Fe}_{2} \mathrm{O}_{3}$ & $\mathrm{CaO}$ & $\mathrm{MgO}$ & $\mathrm{TiO}_{2}$ & $\mathrm{Na}_{2} \mathrm{O}$ & $\mathrm{K}_{2} \mathrm{O}$ & $\mathrm{MnO}_{2}$ \\
\hline Content (\%) & 83.08 & 5.95 & 3.39 & 0.44 & 0.40 & 0.30 & 0.043 & 0.26 & 0.41 & 0.0097
\end{tabular}


GO. The illustration for the fabrication process can be seen in Fig. 1.

2.2.1 Fabrication of GO. Natural microcrystalline graphite oxide was prepared by the oxidation of NMGM powder using a modified Hummers method. ${ }^{31}$ In brief, NMGM powder $(1.5 \mathrm{~g})$ was first mixed with concentrated $\mathrm{H}_{2} \mathrm{SO}_{4}(40 \mathrm{~mL})$ and $\mathrm{NaNO}_{3}$ $(1.5 \mathrm{~g})$ in an ice bath for 1 hour. Then, $\mathrm{KMnO}_{4}(4.5 \mathrm{~g})$ was slowly added to the solution with stirring for 2 hours. Successively, the reaction system was transferred to a $38{ }^{\circ} \mathrm{C}$ oil bath and vigorously stirred for 20 hours, turning the color of the solution from black to dark green. Under vigorous stirring, $150 \mathrm{~mL}$ distilled water was added drop by drop, then the solution was stirred for 30 minutes at $97{ }^{\circ} \mathrm{C}$ and followed by a slow addition of $20 \mathrm{~mL}$ $\mathrm{H}_{2} \mathrm{O}_{2}$. The color of the solution was brown. The residue was washed with $5 \% \mathrm{HCl}$ aqueous solution to remove metal ions and centrifugal washed with distilled water. The washed solution was dispersed to obtain GO solution, this dispersion was sonicated using a Fisher Scientific FS60 ultrasonic bath cleaner $(100 \mathrm{~W})$ for 60 minutes at room temperature.

2.2.2 Fabrication of RGO. RGO was fabricated by solvothermal and chemical reduced method respectively, both of which use GO as the raw material. For solvothermal reduced method, PVP (0.05 g) was added to the low concentration GO solution $\left(\sim 3 \mathrm{mg} \mathrm{mL} \mathrm{mL}^{-1}, 50 \mathrm{~mL}\right)$ with vigorously stirring for an hour. The solution was then solvothermally treated in a Teflonlined autoclave at $180{ }^{\circ} \mathrm{C}$ for 24 hours to form an intermediate solid. This intermediate solid was freeze-dried at $-50{ }^{\circ} \mathrm{C}$ for 12 hours to obtain rubber like SRGO. In addition, $\mathrm{NaOH}$ was added to the low concentration GO solution $\left(\sim 3 \mathrm{mg} \mathrm{mL}^{-1}\right)$ with stirring for 2 minutes until the $\mathrm{pH}$ was 10 . Then, $\mathrm{NaBH}_{4}(4.5 \mathrm{~g})$ was added to the GO solution $(200 \mathrm{~mL})$, and the solution was stirred for 8 hours at $80{ }^{\circ} \mathrm{C}$. After the reaction mixture cooled, $5 \% \mathrm{HCl}$ aqueous solution was added till the $\mathrm{pH}=7$. The solution was repeatedly centrifugal washed with distilled water to obtain RGO solution, and the solution was freeze-dried to obtain reduced graphene oxide powder marked as CRGO.

\subsection{Instruments and characterizations}

The field emission scanning electron microscopy (FE-SEM) and transmission electron microscopy (TEM) images were obtained with the TESCAN MIRA3 LMU type and JEM-2100F type, respectively. The usage of the TESCAN MIRA3 LMU type scanning electron microscope is equipped with Oxford X-Max20 Energy spectrum system. The atomic force microscopic (AFM) images of GNs were measured using a scanning probe microscope (PicoPLws, Molecularlmaging). The samples used for SEM, TEM and AFM characterizations were deposited on silicon wafers, mica sheets and copper mesh and screen, respectively. Fourier transform infrared spectroscopy-attenuated total reflectance (FTIR-ATR) spectra were recorded on a Fourier transform infrared spectrometer (WQF-510A). X-ray diffraction (XRD) patterns were recorded on $\mathrm{D} / \mathrm{max} 2550 \mathrm{VB}^{+} \mathrm{X}$-ray diffractometer using $\mathrm{Cu} \mathrm{K} \alpha$ radiation $(k=0.154178 \mathrm{~nm})$ with $40 \mathrm{kV}$ scanning voltage, $40 \mathrm{~mA}$ scanning current and scanning range from $5^{\circ}$ to $80^{\circ}$. Raman spectra were recorded on a LabRAM HR800 multichannel confocal microspectrometer with $514.5 \mathrm{~nm} \mathrm{Ar}_{2}$ laser excitation. X-ray photoelectron spectra (XPS) were measured on a K-Alpha 1063 photoelectron spectrometer using $\mathrm{Al} \mathrm{K} \alpha(h \nu 1486.6 \mathrm{eV}) \mathrm{X}$-ray as the excitation source. A fourprobe resistivity meter (RTS-8, Four-probe TECH, China) was used to measure the electrical resistivity of the samples.

\section{Results and discussion}

Our strategy for improving comprehensive utilization and broadening usage of NMGM is different from the published ones since producing high-quality graphene from low cost graphite minerals does not involve serious environmental pollution and complicated purification process. For example, highly toxic hydrogen fluoride (HF) was often adopted to remove the complex silicate impurities of the minerals to purify NMGM at the cost of sacrificing the environment. ${ }^{16}$ The advantages of the liquid oxidation-reduction method is that the

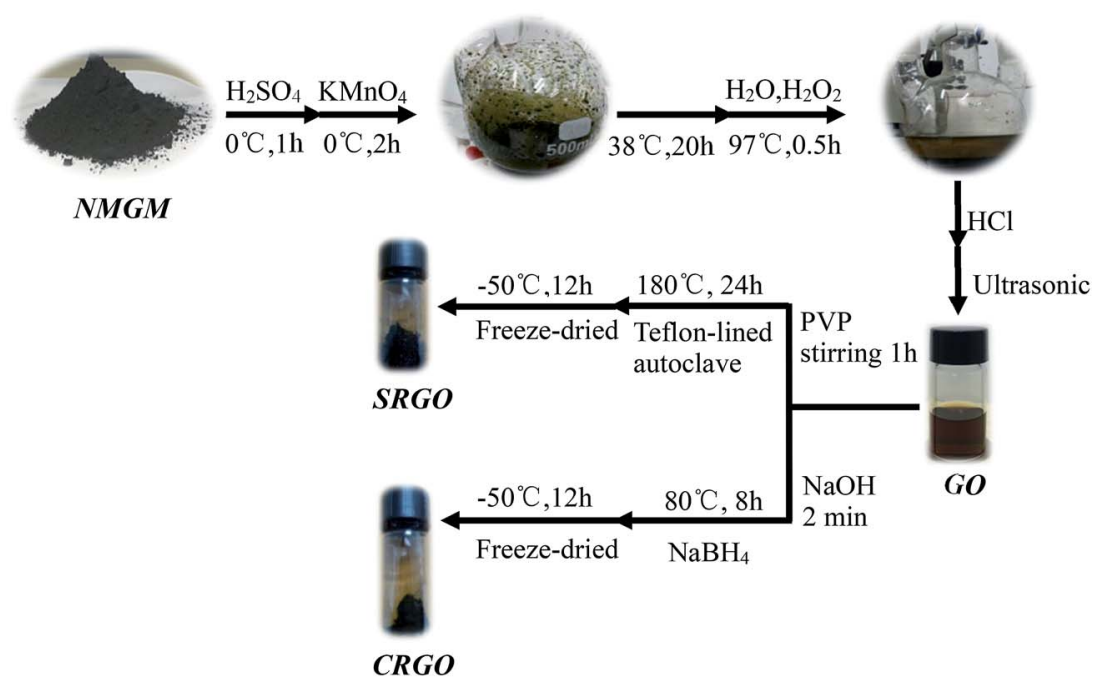

Fig. 1 The preparation process of GNs using NMGMs as the raw material. 
reduction process enable the defects to be repaired and oxygencontaining groups to be removed, and $\pi$-conjugation to be reestablished, compared with the electrochemical \& mechanical exfoliation, low-temperature exfoliation and ball milling method preparing GNs from NMGM. The as-produced GNs can be easily transferred into well distributed bulk materials by freeze-dried approach for further processing and thus applications. We believe these are the reasons the present fabrication can produce graphene sheets with excellent qualities in terms of sizes, purity, and morphology.

We have found that the size of the GNs produced from NMGM is around $1 \mu \mathrm{m} \times 2 \mu \mathrm{m}$, which is larger than the GNs from FGs. The size of graphene sheets were characterized with SEM. The morphologies of NMGM, GO, SRGO, and CRGO were analyzed by SEM (Fig. 2). As seen in Fig. 2(a), when SEM photo is enlarged by 50000 times, the image shows that microscopic particles of NMGM are in laminar shape, and larger particles are composed of tiny laminar particles, and the smaller particles of graphite are absorbed onto the surface of the larger sized graphite particles with arbitrary direction. The smaller graphite particle, after oxidation, is stripped from the larger sized graphite particle. The thick layer graphite is ripped from the flake with curled edges, and the lateral dimensions of the flakes are about $1 \mu \mathrm{m} \times 2 \mu \mathrm{m}$, as shown in Fig. 2(b). Fig. 2(c) shows that the tiny GO flakes are connected with each other to present a random network structure, and the curled edges become less than GO after solvothermal reduction. As seen in Fig. 2(d), the area of CRGO flakes becomes larger and the edge is much curlier when using $\mathrm{NaBH}_{4}$ as a reducing agent. The lateral dimensions of the obtained GNs are about $1 \mu \mathrm{m} \times 2 \mu \mathrm{m}$. Compared with GO, the thickness of CRGO has been increased since the graphene layers reestablished an orderly stack due to the removal of oxidized functional groups. ${ }^{32,33}$ Fig. $\mathrm{S} 1 \dagger$ also show the SEM photo of CRGO with different area. As we all know, the sheet size of GNs prepared from NMGM is smaller than that of GNs prepared from FG. The sheet size of GNs obtained by liquid oxidation-reduction is larger than that of GNs prepared from NMGM by electrochemical \& mechanical exfoliation, low-temperature exfoliation and ball milling. These results all indicate that graphene sheets with up to $1 \mu \mathrm{m} \times 2 \mu \mathrm{m}$ were fabricated from NMGM by liquid oxidation-reduction method.

TEM and AFM clearly show that GNs consist of thin graphene-based sheets on the nanoscale. High-resolution TEM images of NMGM, GO, SRGO and CRGO present the enlarged wrinkles of graphene layers, shown in Fig. 3. Analysis of the edges of graphene in TEM shed light on the thickness of the graphene layers. The layer thickness, which was more than fifty
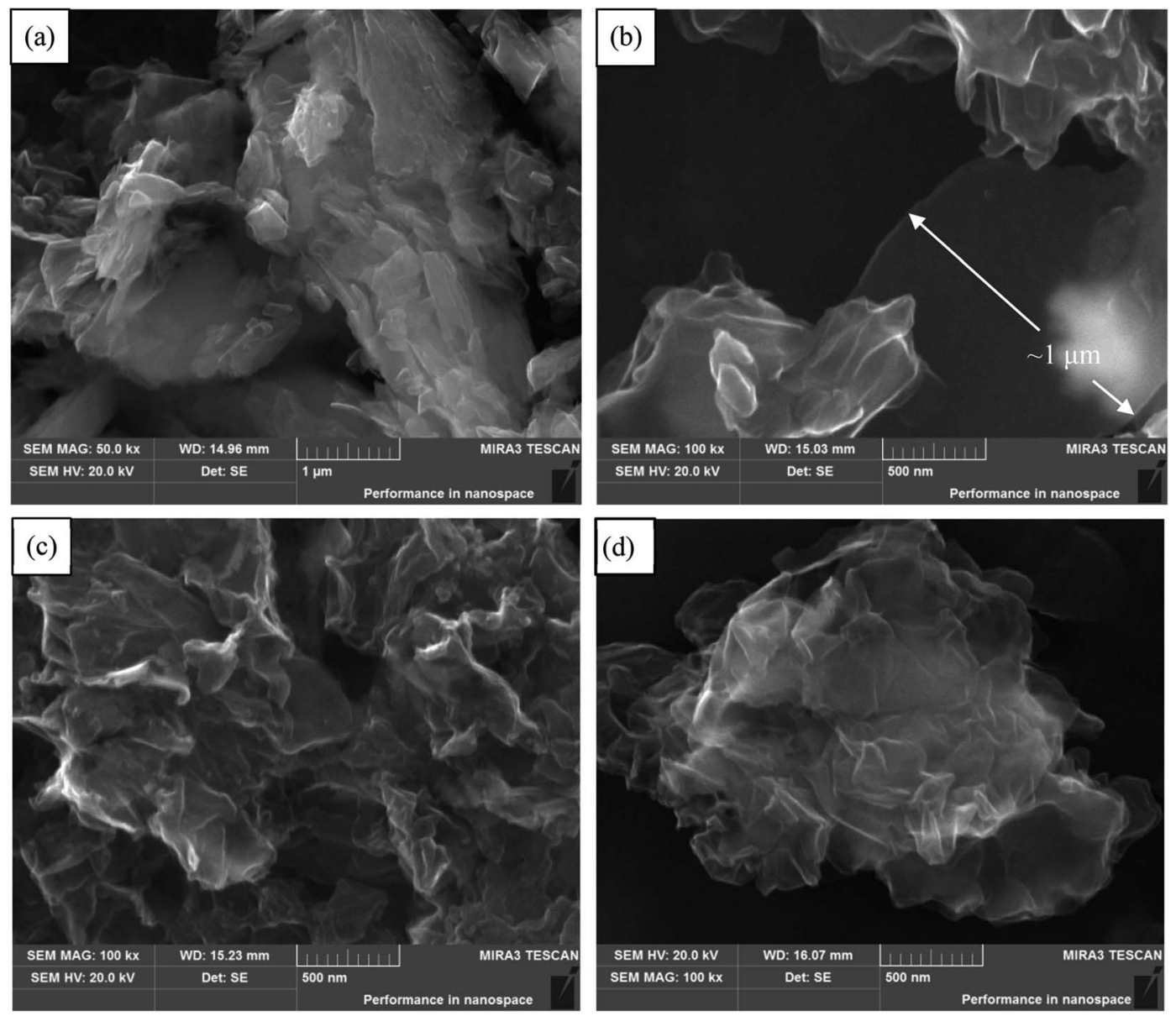

Fig. 2 SEM images of (a) NMGM, (b) GO, (c) SRGO and (d) CRGO. 

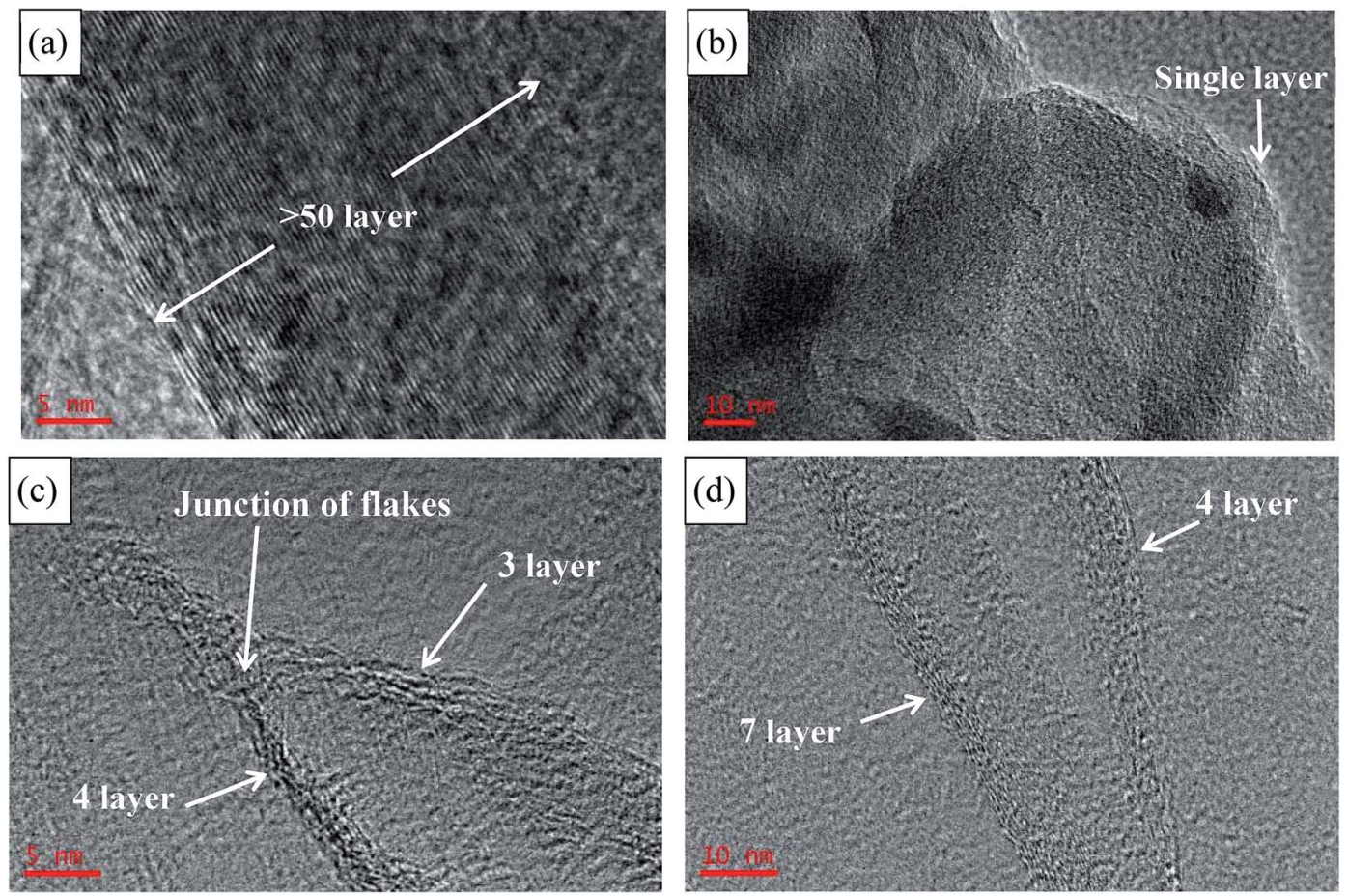

Fig. 3 TEM images of (a) NMGM, (b) GO, (c) SRGO and (d) CRGO.

layers in the NMGM, decreased to single layer after oxidation. The number of layer increased slightly but was still approximately 3-7 wrinkled monolayer graphene after reduction, and it can be seen clearly in Fig. 3(c) that the atoms were not arranged layer by layer at the junction of flakes. The reason for the increasing of layers has been explained in the discussion of SEM. The low-magnification TEM images of samples (Fig. S2 $\dagger$ ) indicate that these ultrathin nanosheets have wrinkled paperlike structures. AFM characterization has been one of the most direct methods of measuring the degree of exfoliation to graphene level after the dispersion of the powder in a solvent. The detailed thickness of GO, SRGO and CRGO sheets were measured by AFM, and Fig. 4(a)-(d) present AFM images of low concentrate NMGM, GO, SRGO and CRGO ethanol solution with ultrasonic dispersed for 2 hours, respectively. NMGM is granular and has a particle size of several hundred nanometers. The thickness of GO sheet is $\sim 0.7 \mathrm{~nm}$, indicating the GO sheet is a single layer graphene oxide, as suggested by previous research. ${ }^{34}$ The thickness of SRGO sheet and CRGO sheet, are $\sim 1.1 \mathrm{~nm}$ and $\sim 2.4 \mathrm{~nm}$, and the surface size of them are larger than GO sheet. Also, the defects which are probably formed by connection of graphene sheets and PVP molecules can be seen in Fig. 4(b), which agree well with the analysis results of SEM and TEM images. ${ }^{35}$

Fewer layers, larger size, and thinner thickness are the important features to measure the quality of graphene. Combined with SEM, TEM and AFM analysis, few-layers $(<7$ layers) GNs with sizes up to $1 \mu \mathrm{m} \times 2 \mu \mathrm{m}$ and $\sim 1.1 \mathrm{~nm}$ thickness were fabricated from NMGM by liquid oxidation-reduction method. In order to gain further insight into the chemical structure changes, FTIR was employed to analyze the existence of most functional groups. Fig. 5 shows the FTIR spectra of GO, SRGO, and CRGO, and Table 2 summarizes the peak positions and their assignments. The peaks of GO centered at 1050, 1220 (1320), 1620,1720 , and $3400 \mathrm{~cm}^{-1}$ were attributed to the $\mathrm{C}-\mathrm{O}-\mathrm{C}$, $\mathrm{C}-\mathrm{OH}, \mathrm{C}=\mathrm{C}, \mathrm{C}=\mathrm{O}, \mathrm{O}-\mathrm{H}$ functionalities, respectively, which are consistent with their characteristic vibration absorption peaks. ${ }^{36}$ After reduction, the peaks for all of the oxygencontaining groups become weak or nearly absent for SRGO and CRGO compared with GO, indicating massive elimination of the oxygen-containing groups during the reduction treatment.

The purity of the GNs product compared with the NMGM was also analyzed by elemental analysis, SEM with energy dispersive X-ray spectrometry (EDS), as shown in Fig. S3 and S4. $\dagger$ Elemental analysis showed that the carbon content of the NMGM is around $49.84 \mathrm{wt} \%$ accompanying with $50 \mathrm{wt} \%$ of other elements such as $\mathrm{Al}, \mathrm{Si}$ and $\mathrm{O}$ (silicate/silica). The carbon content of GNs product from NMGM increases to $60.48 \mathrm{wt} \%$. Furthermore, XPS was further used to probe the chemical compositions of NMGM and GNs sample. Existence of the oxygen functional groups and nitrogen doping and other elements were also confirmed by XPS. Full spectrum in Fig. 6(a) indicates the existence of $\mathrm{Al}, \mathrm{Si}, \mathrm{C}, \mathrm{O}$ and $\mathrm{Na}$ elements in NMGM. Compared to the original ore, the $\mathrm{Al}_{2 \mathrm{p}}$ and the $\mathrm{Si}_{2 \mathrm{p}}$ disappear and the intensities of $\mathrm{C}_{1 \mathrm{~s}}$ and $\mathrm{O}_{1 \mathrm{~s}}$ in the GO, SRGO and CRGO are seriously weakened, suggesting the removal of impurities in the fabrication process involving oxidation and reduction. The reduction effect can be quantitatively characterized by the atomic ratio of carbon to oxygen (Table 3). After oxidation, the $\mathrm{C} / \mathrm{O}$ ratio decreased from 4.5 for NMGM to 1.4 for GO, manifesting the introduction of massive oxygen-containing 


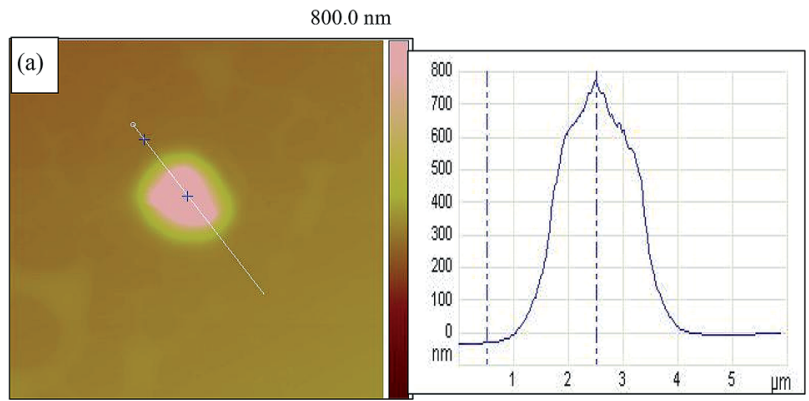

$10.0 \mathrm{~nm}$

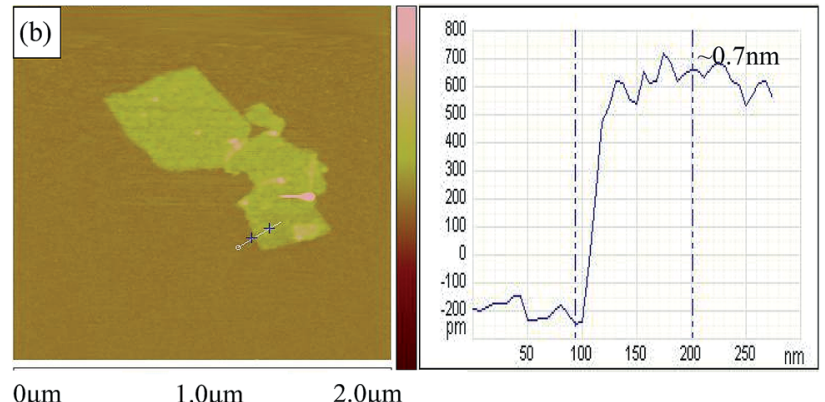

$10.0 \mathrm{~nm}$

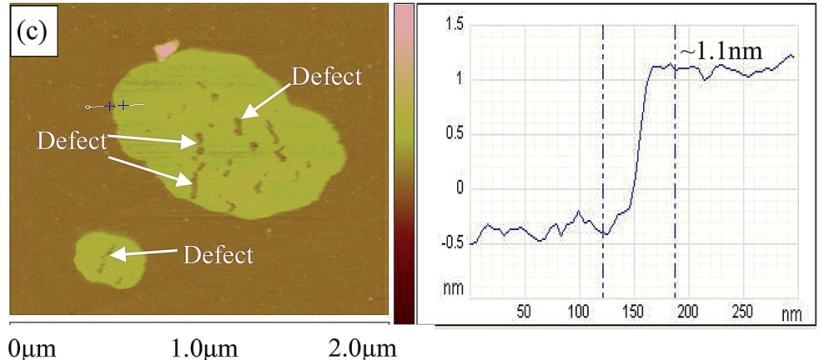

$10.0 \mathrm{~nm}$

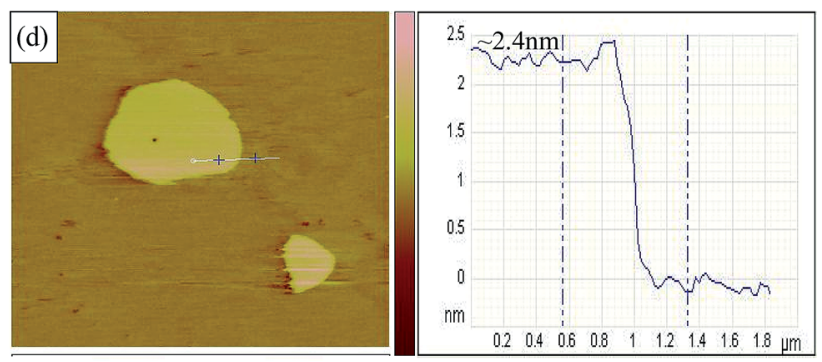

$0 \mu \mathrm{m}$

$1.0 \mu \mathrm{m}$

$2.0 \mu \mathrm{m}$

Fig. 4 AFM images of (a) NMGM, (b) GO, (c) SRGO and (d) CRGO.

functional groups in GO. After reduction, the $\mathrm{C} / \mathrm{O}$ ratio increased from 1.4 for GO to 6.5 for SRGO, which is higher than that of CRGO (3.4).

The high-resolution $\mathrm{C}_{1 \mathrm{~s}}$ spectra of NMGM were deconvoluted into four different peaks that correspond to carbon atoms assuming different binding states [Fig. 6(b)-(e)]. The peaks centered at $284.7,285.5$ and $285.7 \mathrm{eV}$ can be assigned to $\mathrm{C}-\mathrm{C}$ functional groups, $289 \mathrm{eV}$ to $\mathrm{O}-\mathrm{C}=\mathrm{O}$ functional groups. In the case of $\mathrm{C}_{1 \mathrm{~s}}$ spectra of $\mathrm{GO}$, four peaks can be fitted, corresponding to the $\mathrm{sp}^{2} \mathrm{C}=\mathrm{C}$ at $283.3 \mathrm{eV}, \mathrm{C}-\mathrm{C}$ at $284.8 \mathrm{eV}, \mathrm{C}-\mathrm{O}$ at $286.8 \mathrm{eV}$ and $\mathrm{C}=\mathrm{O}$ at $288.3 \mathrm{eV}$. The attendance of these peaks

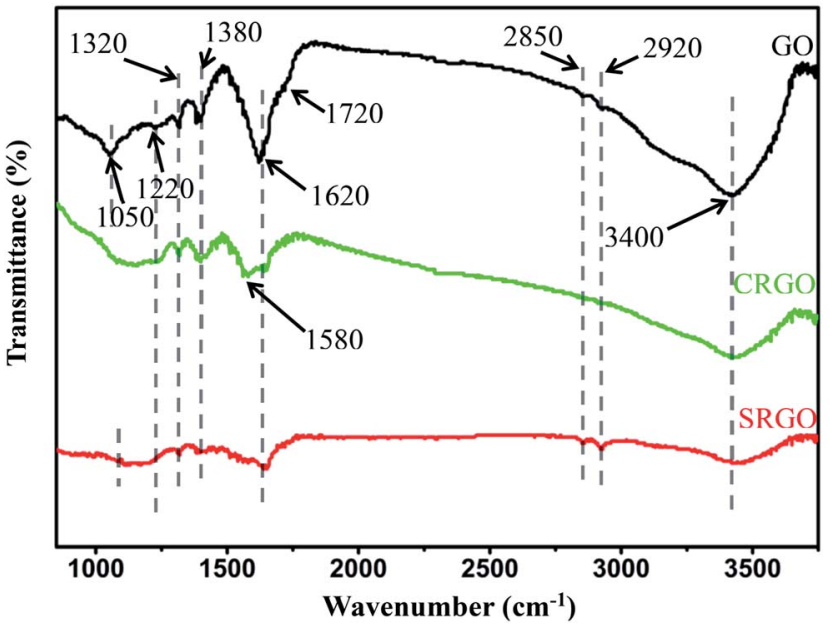

Fig. 5 FTIR spectra of GO, CRGO, SRGO.

indicate that various types of oxygen functionalities exist in the structure of GO. In contrast, the $\mathrm{C}_{1 \mathrm{~s}}$ spectra of SRGO revealed that the intensities of the bands corresponding to oxygencontaining groups were dramatically reduced, confirming the success of the reduction process. The peaks centered at 284.7, 285.9 and $287.2 \mathrm{eV}$ can be assigned to the $\mathrm{C}-\mathrm{C}, \mathrm{C}-\mathrm{N}, \mathrm{C}-\mathrm{O}-\mathrm{C}$ functional groups, respectively. The $\mathrm{C}_{1 \mathrm{~s}}$ spectra of CRGO indicated that the $\mathrm{C}-\mathrm{O}$ almost disappeared and most of $\mathrm{C}=\mathrm{O}$ were removed effectively. The peaks centered at 283.6 and $285.7 \mathrm{eV}$ can be assigned to the $\mathrm{sp}^{2} \mathrm{C}=\mathrm{C}$ and $\mathrm{C}-\mathrm{C}$ functional groups. ${ }^{37-41}$ $\mathrm{XPS}_{1 \mathrm{~s}}$ spectra in Fig. S5 and Table $\mathrm{S} 1 \dagger$ depict the detail of the oxygen functionalities for NMGM, GO, SRGO and CRGO. It can be concluded that the oxidation, and exfoliation and reduction processes have changed the number of surface species. The results of $\mathrm{O}_{1 \mathrm{~s}}$ spectra are corroborated by $\mathrm{C}_{1 \mathrm{~s}}$ spectra.

The full XPS spectra for SRGO shows a distinguished $\mathrm{N}$ signal at about $400.0 \mathrm{eV}$ [Fig. 6(a)], confirming the nitrogendoping of graphene. The $\mathrm{N}_{1 \mathrm{~s}}$ spectrum of SRGO exhibits only one peak at $\sim 400.0 \mathrm{eV}$, and deconvolution of the $\mathrm{N}_{1 \mathrm{~s}}$ spectra [Fig. 6(f)] for the exhausted samples shows two peaks with their binding energy equal to $399.8 \mathrm{eV}$ and $401.9 \mathrm{eV}$. The first peak represents nitrogen involved in $\mathrm{C}-\mathrm{N}$ (from amines or amides), whereas the second peak can be assigned to $\mathrm{C}-\mathrm{N}^{+}$(from quaternary nitrogen) or $\mathrm{NH}_{4}{ }^{+}$, further demonstrating that the nitrogen atoms came from PVP.

It is obvious that the process of GNs fabricated from NMGM using liquid oxidation-reduction method can be divided into oxidation, stripping, and reduction stages. As shown in Tables 2 and 3, analyses from both FTIR and XPS clearly suggest that considerable changes of oxygen-containing groups, chemical states and element compositions, such as $\mathrm{C}, \mathrm{Al}, \mathrm{Si}$ and $\mathrm{O}$ (complex silicate and silica) occurred in the three stages. The $\mathrm{C} /$ $\mathrm{O}$ atomic ratios for raw mineral and GO calculated from the corresponding peak areas of the XPS are determined as 4.5 and 1.4, respectively, showing a complete oxidation degree of the GO. The reason is that, in the oxidation stage of NMGM, oxidation attack initiates on the carbon atoms at the edges or defects of NMGM, forming the hydroxyl groups. With the 
Table 2 FTIR spectra of GO, SRGO, and CRGO

\begin{tabular}{llll}
\hline Assignment & GO & SRGO & CRGO \\
\hline C-O-C str vibr & 1050 & 1060 & - \\
C-OH (COOH) & 1220,1320 & 1220,1320 & 1220,1320 \\
str vibr & & & \\
C=C str vibr & 1620 & 1630 & 1580,1620 \\
C=O str vibr & 1720 & - & 1710 \\
C-H bend vibr/ & $1380 / 2850,2920$ & $1380 / 2850,2920$ & $1380 / 2850,2920$ \\
str vibr & & 3420 & 3400 \\
O-H str vibr & 3400 & & \\
\hline
\end{tabular}

progress of oxidation, more hydroxyl groups are generated on the basal planes. Simultaneously, the formed hydroxyl groups at the edges are continuously oxidized to the carbonyl groups in the forms of the ketone and/or quinone groups, and the neighboring hydroxyl groups on the basal planes condense to the epoxy groups by dehydration in the strong acid environment. The relative atomic concentrations of $\mathrm{C}-\mathrm{O}$ and $\mathrm{C}=\mathrm{O}(\mathrm{O}-$ $\mathrm{C}=\mathrm{O}$ ) in $\mathrm{GO}$ is $25.39 \%$ and $20.19 \%$, indicating the complete oxidation of the edges that form hydroxyl and carbonyl groups. Then, the ketone groups at the edges are further oxidized to the carboxyl groups with the six-member carbon ring opening in the presence of excess $\mathrm{KMnO}_{4}$ and concentrated $\mathrm{H}_{2} \mathrm{SO}_{4}{ }^{42}$ Therefore, the oxygen-containing groups of NMGM oxide formed in the oxidation stage.

Solvothermal and chemical reduction strategy were used to obtain GNs. The C/O atomic ratios of SRGO is 6.5, which is significantly higher than those of the raw minerals and RGO. ${ }^{27,28}$ The relative atomic concentrations of $\mathrm{C}-\mathrm{O}-\mathrm{C}$ and $\mathrm{C}-\mathrm{C} / \mathrm{C}-\mathrm{N}$ in SRGO is $13.72 \%$ and $20.20 \%$. Results show that oxygencontaining groups of GO were remarkably deoxygenated owing to their poor thermal stability in the solvothermal reduction reactions. During the reduction stage of GO, we tentatively speculate the deoxygenation reaction as follow. First, autogenic pressure at $180{ }^{\circ} \mathrm{C}$ facilitates large amounts of free hydrogen radicals divorced from PVP, which is a dehydrogenation reaction process. Then, the deoxygenation of epoxide, carbonyl, carboxyl and hydroxyl on GO is promoted by the high temperature and the self-generated pressure in the sealed reaction autoclave. The superheated PVP under the hydrothermal conditions behaves like a strong electrolyte with a high diffusion coefficient and dielectric constant, and facilitates the bond cleavage of oxygen functional groups. ${ }^{41,43}$ The subsequent annealing step would remove most of the functional groups, partially restoring $\pi$-conjugation. Besides, the introduction of PVP can assemble the wonderful 2D graphene sheets into bulk 3D state, which further confirmed random network structure in Fig. 2(c). In addition, $\mathrm{NaBH}_{4}$, a common reducing agent, can reduce carboxyl, carbonyl and epoxy groups into hydroxyl group. However, it is difficult to eliminate the hydroxyl group. ${ }^{41}$ The C/O of CRGO (3.4) also confirmed this mechanism. Obviously, solvothermal reduction is more effective than that of $\mathrm{NaBH}_{4}$.

We also monitored the structural deformation during the preparation process of GNs by X-ray diffraction, which
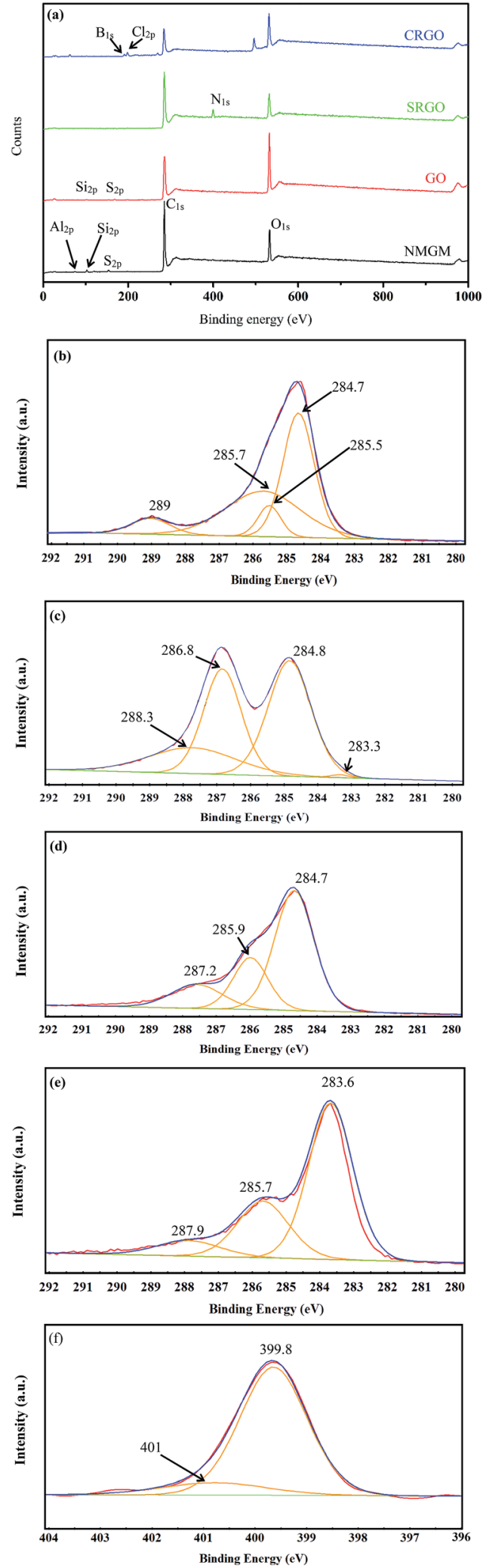

Fig. 6 XPS spectra of NMGM, GO, CRGO and SRGO (a) and $C_{1 s}$ XPS spectra of NMGM (b), GO (c), CRGO (d) and SRGO (e) and $N_{1 s}$ XPS spectrum of SRGO (f). 
Table 3 Chemical states of $\mathrm{C}$ and $\mathrm{O}$ atoms on NMGM, GO, SRGO, and CRGO with their relative atomic concentrations (atom\%)

\begin{tabular}{|c|c|c|c|c|c|c|c|c|}
\hline Samples & $\mathrm{C}(\mathrm{at} \%)$ & $\mathrm{O}(\mathrm{at} \%)$ & $\mathrm{C} / \mathrm{O}$ & $\mathrm{C}-\mathrm{C} / \mathrm{C}=\mathrm{C}(\mathrm{at} \%)$ & $\mathrm{C}-\mathrm{O}(\mathrm{at} \%)$ & $\mathrm{C}=\mathrm{O}(\mathrm{O}-\mathrm{C}=\mathrm{O})(\mathrm{at} \%)$ & $\mathrm{C}-\mathrm{O}-\mathrm{C}(\mathrm{at} \%)$ & $\mathrm{C}-\mathrm{C} / \mathrm{C}-\mathrm{N}(\mathrm{at} \%)$ \\
\hline NMGM & 77.94 & 17.36 & 4.5 & 92.85 & - & 7.15 & - & - \\
\hline GO & 57.44 & 40.93 & 1.4 & 44.41 & 25.39 & 20.19 & - & - \\
\hline CRGO & 67.71 & 19.95 & 3.4 & 90.82 & - & 9.18 & - & - \\
\hline
\end{tabular}

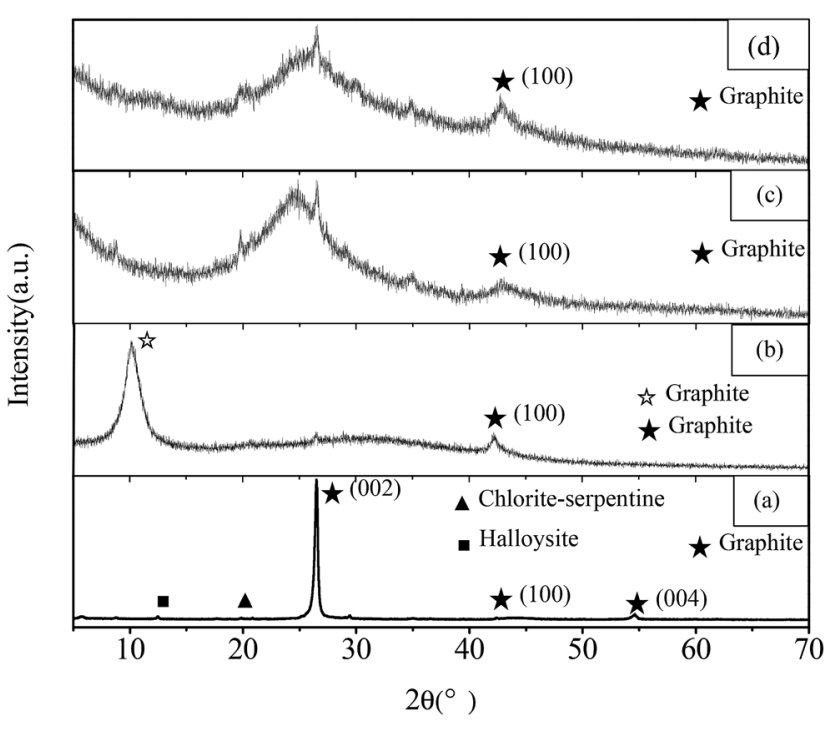

Fig. 7 XRD patterns of (a) NMGM, (b) GO, (c) SRGO and (d) CRGO.

demonstrates that amorphous structure is achieved during the fabrication process of GNs from NMGM. The powder XRD patterns of NMGM, GO, SRGO and CRGO are presented in Fig. 7. The (002), (100) and (004) diffraction peaks of graphite in Fig. 7(a) reveal that the NMGM has a typical graphite- $2 \mathrm{H}$ structure. The calculation of the NMGM particle size by Scherrer formula $[D=K \gamma / B \cos \theta$, for the C (002) crystal plane reflection peak] gives the value of $23.56 \mathrm{~nm}$. It can be deduced

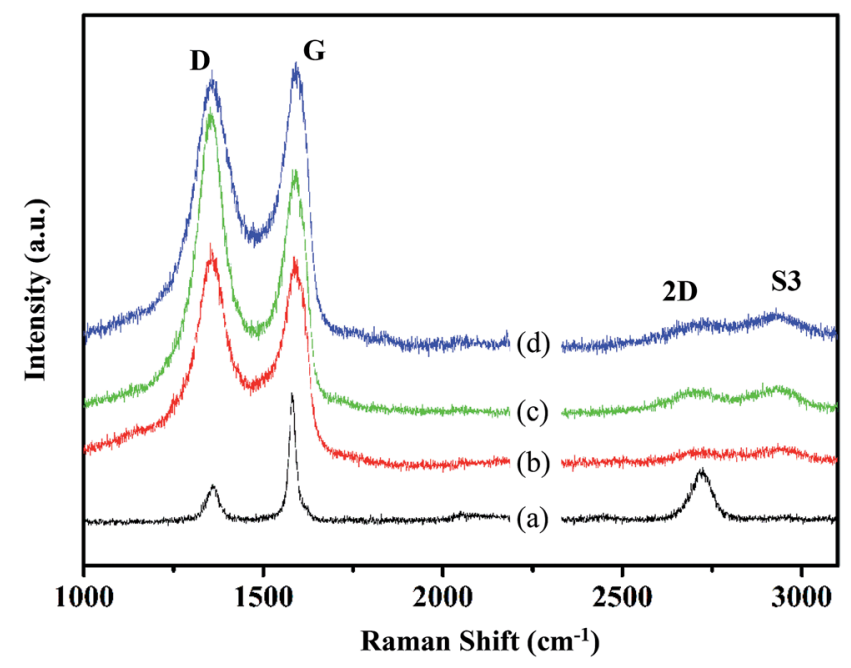

Fig. 8 Raman spectra of (a) NMGM, (b) GO, (c) SRGO and (d) CRGO. that the NMGM consists of 69 atom layers (a single layer of carbon atom in a closely packed honeycomb two dimensional lattice is about $0.34 \mathrm{~nm}$ ). The results of XRD are in agreement with the SEM and TEM analyses. Besides, the NMGM ore contains impurities such as halloysite and chlorite-serpentine. The disappearance of the graphite peaks and the appearance of the graphite oxide peak $\left(2 \theta=9.5^{\circ}\right)$ in XRD pattern of GO [Fig. 7(b)], demonstrates that the graphite is oxidated completely. In Fig. 7(b) and (c), the disappearance of the graphite (002) peak shows that the stacking structure of the graphite layer has disappeared, which indicates that the graphite layer has been stripped away. Compared with Fig. 7(b)(d) show that the graphite oxide peak, as indicated by the broad peak between $2 \theta$ of $20^{\circ}$ and $30^{\circ}$, which is the (100) diffraction peaks of graphite. .445 $^{4}$

Raman spectroscopy was used to obtain more information about the structure of the synthesized graphene. The Raman spectra of NMGM, GO, SRGO and CRGO are associated with a typical $\mathrm{D}$ band and $\mathrm{G}$ band around $1360 \mathrm{~cm}^{-1}$ and $1580 \mathrm{~cm}^{-1}$, respectively (as shown in Fig. 8). The D band is due to the breathing mode of $\kappa$-point photons of $A_{1 \mathrm{~g}}$ symmetry, and the $\mathrm{G}$ band is due to the first-order scattering of $E_{2 g}$ phonons by $\mathrm{sp}^{2}$ carbon atoms. The presence of the D-band indicates defects in

Table 4 D/G ratios and the corresponding crystallite sizes of the samples measured by Raman spectroscopy

\begin{tabular}{lrr}
\hline Samples & $I_{\mathrm{D}} / I_{\mathrm{G}}$ & $L_{\mathrm{a}}(\mathrm{nm})$ \\
\hline NMGM & 0.239 & 4.004 \\
GO & 1.280 & 21.442 \\
SRGO & 1.420 & 23.788 \\
CRGO & 1.150 & 19.265
\end{tabular}

Table 5 Electrical resistivity of the samples

\begin{tabular}{lllll}
\hline Sample & NMGM & GO & SRGO & CRGO \\
\hline Resistivity $(\Omega \mathrm{cm})$ & 7.1 & $0.76 \times 10^{5}$ & 3.2 & 9.0
\end{tabular}

Table 6 Weight of graphene in each $5 \mathrm{~mL}$ dispersion

\begin{tabular}{lllllllllll}
\hline Samples & 1 & 2 & 3 & 4 & 5 & 6 & 7 & 8 & 9 & 10 \\
\hline Weight (mg) & 14 & 13 & 13 & 14 & 15 & 14 & 15 & 14 & 14 & 14
\end{tabular}




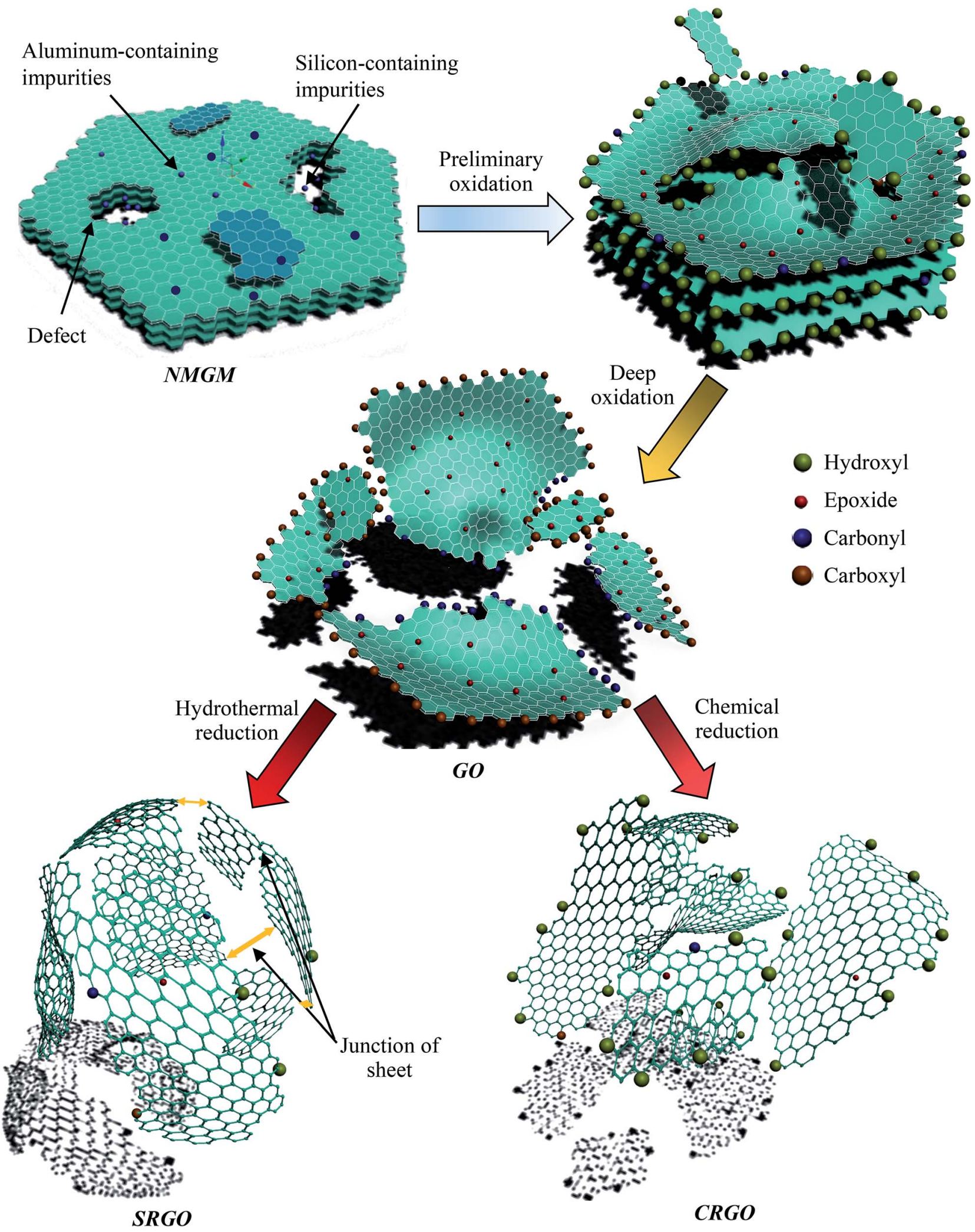

Fig. 9 The schematic structure evolutions of GNs prepared by the liquid oxidation-reduction method from NMGM. The oxidation stage of NMGM. Hydroxyl group, carbonyl and carboxyl group and epoxy group formed in this stage. In general, the electron donating groups (i.e., hydroxyl and epoxy) form before the electron withdrawing groups (i.e., the carbonyl and carboxyl groups). It is important to note that the formation process of carboxyl groups is relatively slower compared with that of the carbonyl groups, because the oxidant is consumed by the precedent fast oxidation process. Parallel to the sequential oxidation is the dehydration that forms the epoxy groups once the neighboring hydroxyl groups emerge. ${ }^{37}$ Moreover, metal oxides $\left(\mathrm{Fe}_{2} \mathrm{O}_{3}, \mathrm{CaO}, \mathrm{K}_{2} \mathrm{O}, \mathrm{Na}_{2} \mathrm{O}, \mathrm{TiO}_{2}, \mathrm{MgO}\right.$ and $\mathrm{MnO}$ ) in the $\mathrm{NMGM}$ would react with $\mathrm{H}_{2} \mathrm{SO}_{4}$ and most of metallic oxides in complicated silicate minerals were 
the graphene layer, primarily attributed to the presence of $\mathrm{sp}^{3}$ bonded carbon atoms. The G-band resulted from $\mathrm{sp}^{2}$-bonded carbon atoms in the graphene layer. Furthermore, the average crystallite size $\left(L_{\mathrm{a}}\right)$ of the defect-free domains within the graphene was calculated using the equation ${ }^{43,46,47}$

$$
L_{\mathrm{a}}=2.4 \times 10^{-10} \times \lambda_{\text {laser }}^{4} \times I_{\mathrm{G}} / I_{\mathrm{D}}
$$

where $I_{\mathrm{G}} / I_{\mathrm{D}}$ (where the D peak is a defect peak due to intervalley scattering and $\mathrm{G}$ refers to the graphene $\mathrm{G}$ peak, the $\mathrm{S} 3$ peak is a second-order peak due to the $\mathrm{D}-\mathrm{G}$ combination, and $2 \mathrm{D}$ refers to the graphene 2D peak) is the ratio of the intensities of the Dand G-band, respectively, and $\lambda_{\text {laser }}$ refers to the laser wavelength $(\mathrm{nm})$ used in the measurement of the Raman spectrum. The calculated $\mathrm{D} / \mathrm{G}$ ratios and the corresponding crystallite sizes are compiled in Table 4. After oxidation, the Raman D/G intensity ratios increase from 0.239 in NMGM to 1.280 in GO, which is caused by addition of the oxygen functional group. During the solvothermal reduction process, most of the oxygencontaining functional groups have been removed from the carbon layer, the defects in the carbon lattice structure have been aroused which allow the value of $I_{\mathrm{D}} / I_{\mathrm{G}}$ increase from 1.280 in GO to 1.420 in SRGO. However, after the chemical reduction process, the value of $I_{\mathrm{D}} / I_{\mathrm{G}}$ is smaller than SRGO, which is due to the ordered stacking of reduced graphene oxide sheets. This clearly indicates a significant influence of graphite oxide preparation method on graphene structure and defect density. A slightly higher $\mathrm{D} / \mathrm{G}$ ratio is observed for samples reduced by solvothermal method compared with chemical reduction. XRD and Raman results indicate that the obtained GNs exhibit an amorphous nanostructure.

The electrical properties of the samples are shown in Table 5 . The electrical resistivity of GO is $0.76 \times 10^{5} \Omega \mathrm{cm}$, which is much higher than that of NMGM $(7.1 \Omega \mathrm{cm}), \operatorname{SRGO}(3.2 \Omega \mathrm{cm})$ and CRGO $(9.0 \Omega \mathrm{cm})$. This indicates that lots of oxygencontaining groups were introduced into GO. The results also indicate that solvothermal reduction is more effective in restoring the $\pi$-conjugated structure of graphene than chemical reduction..$^{48}$ Therefore, the electrical properties of GNs can be adjusted by controlling the oxidation degree of NMGM and also the reduction degree of GO.

The disperse and homogeneous graphene dispersion we prepared is $500 \mathrm{~mL}$, taking $5 \mathrm{~mL}$ to freeze drying and the data obtained is shown in Table 6. After taking the arithmetic average, every $5 \mathrm{~mL}$ graphene dispersion contains $14 \mathrm{mg}$ graphene, and the productivity:

$$
\eta=M_{\mathrm{SRGO}} / M_{\mathrm{NMGM}}=14 \mathrm{mg} \times 100 / 1500 \mathrm{mg}=93.3 \% .
$$

In this study, $1.4 \mathrm{~g}$ graphene is fabricated from $1.5 \mathrm{~g}$ graphite.
According to the above investigation, the preparation process can be divided into oxidation, stripping, and reduction stages. Among them, chemical reaction occurs in the oxidation, and reduction stages, while the stripping stage is a physical process. Therefore, the critical stages are the oxidation and reduction process of GO. The schematic structure evolutions of the GNs prepared from NMGM is portrayed in Fig. 9 and S6. $\dagger$

We compare the differences between natural flake graphite (FG) and natural microcrystalline graphite (MG), as well as the graphene oxide (GO) and the reduced graphene oxide (RGO) prepared by the same method. We label the graphene oxide prepared with FG and MG as the raw material with F-GO and MGO, the solvothermal reduced graphene oxide with F-SRGO and $\mathrm{M}$-SRGO, and the chemical reduced graphene oxide with FCRGO and M-CRGO, respectively. In the SEM image and the AFM image (shown in Fig. S6 and S7 $\dagger$ ) comparing FG with MG, it is found that FG crystal has a sheet structure with a particle size of tens of micrometers, while MG has a granular structure with a particle size of several micrometers. Meanwhile, the surface of FG crystal is more smooth and has obvious anisotropy, which directly lead to the difference in the morphology of the graphene prepared. The sheet of F-GO is larger with a lateral dimension of tens of microns, but the thickness of the sheet is much thinner than that of FG. While the lateral dimension of the sheet of M-GO is only a fraction of a micron, and the thickness is thinner than that of F-GO which is only $1.1 \mathrm{~nm}$ with a more obvious edge curl. It can be seen from the figure that the sheet area of the graphene prepared with microcrystalline graphite as the raw material is smaller, and the thickness is thinner with a higher curl degree. From the XRD graph (shown in Fig. 8 and 9) comparing both, it is found that MG contains a variety of impurities, including chlorite-serpentine and halloysit, while the purity of FG graphite is higher. Compared to MG, the peak of the graphite oxide slightly moves to left, and all the graphene prepared produce the broad peak which should be create by the reduced graphene oxide at a temperature near $2 \theta=$ $45^{\circ}$, and The GO and RGO prepared with microcrystalline graphite as the raw material have the (100) diffraction peak of the graphite crystal.

\section{Conclusions}

GNs were fabricated using NMGM instead of FG by liquid oxidation-reduction method. The GNs with layer numbers ranges from 3 to 7 , thickness of $1.1 \mathrm{~nm}$, and sizes up to $1 \mu \mathrm{m} \times 2$ $\mu \mathrm{m}$ are obtained. The graphene products exhibit the features of large amounts of defects and possess an amorphous nanostructure. Most impurities of the NMGM were removed in the oxidation process and the carbon content increased after reduction of GO. The formation mechanism of GNs is mainly

dissolved in the solutions as salt during the treatment. The complicated silicate structures of the impurities were destroyed by the interaction of NMGM with $\mathrm{H}_{2} \mathrm{SO}_{4}$, and the metal oxides in it were removed in form of soluble salts as reactions proceeded. Most of the impurities can be removed by washing with water after the oxidation reaction, but the reaction mainly took place on the interface of the sulphuric acid and graphite particles. The reduction stage of GO. The deoxygenation of epoxide, carbonyl, and hydroxyl on GO is removed by chemicals or the high temperature and the self-generated pressure. Partial explanation has been displayed in the analysis of FTIR and XPS. The complete reduction from GO to GNs is hard to achieve because the GO sheets with a high concentration of defects are difficult to fully deoxygenate and the defects themselves are difficult to heal by post-treatment 
the generation and eliminating of oxygen-containing groups or re-establishing the conjugated graphene network. The GNs products fabricated by both the chemical and solvothermal reduced methods are similar in their dispersibility, chemical structures, thicknesses, and lateral dimensions. Furthermore, the exclusion of chemicals could affect the efficiency of the overall reaction. This facile approach without any purification greatly facilitates preparation of high-quality graphene from abundant graphite minerals for the commercialization of graphene production.

\section{Acknowledgements}

This work is supported by National Natural Science Foundation of China (No. 51201022), China Postdoctoral Science Foundation (No. 2013M542555), the Science \& Technology Program of Changsha (No. K1508005-11), open Fund of Hunan Province Key Laboratory of Safety Design and Reliability Technology for Engineering Vehicle (No. KF1509) and open Research Fund of the Hunan Province Higher Education Key Laboratory of Modeling and Monitoring on the Near-Earth Electromagnetic Environments (No. 20150109) in Changsha University of Science \& Technology, and Hunan Provincial Innovation Foundation For Postgraduate (CX2016B399). W. X. appreciates the great effort of Jiaqi Wang (The University of Tennessee, Knoxville) for the helpful discussion and proofreading for improving the English writing quality of the manuscript.

\section{References}

1 K. Hu, D. D. Kulkarni, I. Choi and V. V. Tsukruk, Prog. Polym. Sci., 2014, 39, 1934-1972.

2 S. Shin and M. Kaviany, Phys. Rev. B: Condens. Matter Mater. Phys., 2011, 84, 4319-4322.

3 L. Ma, H. Niu, J. Cai, P. Zhao, C. Wang, Y. Lian, X. Bai and W. Wang, J. Mater. Chem. C, 2014, 2, 2272-2282.

4 S. Stankovich, D. A. Dikin, R. D. Piner, K. A. Kohlhaas, A. Kleinhammes, Y. Jia, Y. Wu, S. T. Nguyen and R. S. Ruoff, Carbon, 2007, 45, 1558-1565.

5 J. Chen, B. Yao, C. Li and G. Shi, Carbon, 2013, 64, 225-229. 6 A. M. Dimiev and J. M. Tour, ACS Nano, 2014, 8, 3060-3068. 7 S. Dubln, S. Gilje, K. Wang, V. C. Tung, K. Cha, A. S. Hall, J. Farrar, R. Varshneya, Y. Yang and R. B. Kaner, ACS Nano, 2010, 4, 3845-3852.

8 B. Shen, D. Lu, W. Zhai and W. Zheng, J. Mater. Chem. C, 2013, 1, 50-53.

9 C. Nethravathi and M. Rajamathi, Carbon, 2008, 46, 19941998.

10 S. Stankovich, D. A. Dikin, G. H. Dommett, K. M. Kohlhaas, E. J. Zimney, E. A. Stach, R. D. Piner, S. T. Nguyen and R. S. Ruoff, Nature, 2006, 442, 282-286.

11 Y. Wu, N. Yi, L. Huang, T. Zhang, S. Fang, H. Chang, N. Li, J. Oh, J. A. Lee, M. Kozlov, A. C. Chipara, H. Terrones, P. Xiao, G. Long, Y. Huang, F. Zhang, L. Zhang, X. Lepro, C. Haines, M. D. Lima, N. P. Lopez, L. P. Rajukumar, A. L. Elias, S. Feng, S. J. Kim, N. T. Narayanan,
P. M. Ajayan, M. Terrones, A. Aliev, P. Chu, Z. Zhang, R. H. Baughman and Y. Chen, Nat. Commun., 2015, 6, 6141. 12 D. He, Z. Peng, W. Gong, Y. Luo, P. Zhao and L. Kong, RSC Adv., 2015, 5, 11966-11972.

13 Y. Zhang, Y. Huang, T. Zhang, H. Chang, P. Xiao, H. Chen, Z. Huang and Y. Chen, Adv. Mater., 2015, 27, 2049-2053.

14 F. Wu, A. Xie, M. Sun, Y. Wang and M. Wang, J. Mater. Chem. A, 2015, 3, 14358-14369.

15 K. Shen, Z.-H. Huang, K. Hu, W. Shen, S. Yu, J. Yang, G. Yang and F. Kang, Carbon, 2015, 90, 197-206.

16 W. Xie, Z. Wang, J. Kuang, H. Xu, S. Yi, Y. Deng, T. Cao and Z. Guo, Int. J. Miner. Process., 2016, 155, 45-54.

17 S. Li, L. Ma, H. Long, X. Yu, H. Luo, Y. Wang, H. Zhu, Z. Yu, M. Ma and Q. Wei, Appl. Surf. Sci., 2016, 367, 473-479.

18 Y. Wei, Y. Jiang, N. Li, Z. Hu, X. He and X. Ouyang, Adv. Appl. Ceram., 2015, 114, 423-428.

19 H. Wang, Y. Li, T. Zhu, S. Sang and Q. Wang, Ceram. Int., 2014, 40, 11139-11148.

20 H. Wang, Y. Li, S. Sang, S. Jin, Y. Xu, K. Yang and S. Yu, Ceram. Int., 2014, 40, 15783-15793.

21 T. Wenmao, P. Qun, L. Yanyan and W. Nan, Int. J. Electrochem. Sci., 2012, 7, 11578-11587.

22 C. Sole, N. E. Drewett and L. J. Hardwick, Faraday Discuss., 2014, 172, 223-237.

23 K.-J. Kim, T.-S. Lee, H.-G. Kim, S.-H. Lim and S.-M. Lee, Electrochim. Acta, 2014, 135, 27-34.

24 D. Deýlová, V. Vyskočil and J. Barek, J. Electroanal. Chem., 2014, 717-718, 237-242.

25 K. Li, K. Shen, Z.-H. Huang, W. Shen, G. Yang, J. Yang and F. Kang, Fuel, 2016, 180, 743-748.

26 W. Xie, X. Zhu, S. Yi, J. Kuang, H. Cheng, W. Tang and Y. Deng, Mater. Des., 2016, 90, 38-46.

27 J. Wang, J. Huang, R. Yan, F. Wang, W. Cheng, Q. Guo and J. Wang, J. Mater. Chem. A, 2015, 3, 3144-3150.

28 H. Xian, T. Peng, H. Sun and J. Wang, J. Mater. Sci., 2015, 50, 4025-4033.

29 S. F. Lin, F. X. Liu and G. H. Chen, $R S C A d v ., 2014,4$, 4588545889.

30 H. Xian, T. Peng, H. Sun and J. Wang, J. Mater. Sci.: Mater. Electron., 2014, 26, 242-249.

31 W. S. Hummers and R. E. Offeman, J. Am. Chem. Soc., 1958, 80, 1339.

32 Y. Huang, Y. Qin, Y. Zhou, H. Niu, Z. Z. Yu and J. Y. Dong, Chem. Mater., 2010, 22, 4096-4102.

33 C. Liu, Z. Yu, D. Neff, A. Zhamu and B. Z. Jang, Nano Lett., 2010, 10, 4863-4868.

34 G. Shao, Y. Lu, F. Wu, C. Yang, F. Zeng and Q. Wu, J. Mater. Sci., 2012, 47, 4400-4409.

35 S. Pei and H.-M. Cheng, Carbon, 2012, 50, 3210-3228.

36 A. Kumar and M. Khandelwal, New J. Chem., 2014, 38, 3457.

37 J. Zhu, R. Duan, Y. Zhang and J. Zhu, Ceram. Int., 2016, 42, 334-340.

38 P. Dong, Y. Wang, L. Guo, S. Xin, J. Zhang, Y. Shi, W. Zeng and S. Yin, Nanoscale, 2012, 4, 4641-4649.

39 Y. Shen, H.-B. Zhang, H. Zhang, W. Ren, A. Dasari, G.-S. Tang and Z.-Z. Yu, Carbon, 2013, 56, 132-138. 
40 C. Petit, M. Seredych and T. J. Bandosz, J. Mater. Chem., 2009, 19, 9176-9185.

41 C. K. Chua and M. Pumera, Chem. Soc. Rev., 2014, 43, 291312.

42 Z. Liu, X. Duan, X. Zhou, G. Qian, J. Zhou and W. Yuan, Ind. Eng. Chem. Res., 2014, 53, 253-258.

43 Z. Sofer, O. Jankovský, P. Šimek, D. Sedmidubský and J. Šturala, ACS Nano, 2015, 9, 5478-5485.

44 A. Kumar and M. Khandelwal, New J. Chem., 2014, 38, 3457.
45 M. Seredych and T. J. Bandosz, J. Phys. Chem. C, 2007, 111, 15596-15604.

46 V. Barbarossa, F. Galluzzi, R. Tomaciello and A. Zanobi, Chem. Phys. Lett., 1991, 185, 53-55.

47 H. Wang, J. T. Robinson, X. Li and H. Dai, J. Am. Chem. Soc., 2009, 131, 9910-9911.

48 R. Wang, Y. Wang, C. Xu, J. Sun and L. Gao, RSC Adv., 2013, 3, 1194-1200. 